\title{
Mucocele de apêndice: Relato de dois casos e revisão literária
}

\author{
Mucocele of the appendix: Report of two cases and literature review \\ Richard Raphael Borges Tavares Vieira ${ }^{\dagger \S}$, Ana Beatriz Esteves Batista ${ }^{\dagger \S}$, Victor Carlos Saiz Vieira ${ }^{\dagger \S}$, \\ Laura Dias Pereira ${ }^{\star \$}$, Joaquim Ferreira de Paula ${ }^{\star 8}$, Carlos Augusto Marques Batista ${ }^{\star 8}$
}

Como citar esse artigo. Vieira, RRBT; Batista, ABE; Vieira, VCS; Pereira LD; de Paula, JF; Batista, CAM Mucocele de apêndice: Relato de dois casos e revisão literária. Revista de Saúde. 2018 Jan./Jun.; 09 (1): 09-13.

\begin{abstract}
Resumo
A mucocele de apêndice ocorre após sua obstrução quando há acúmulo de material mucinoso em seu interior, dilatando assim sua luz. É uma doença rara e geralmente assintomática, sendo na maioria das vezes um achado incidental no momento da laparotomia. Todavia, pode cursar com dor abdominal, alterações de hábito intestinal, massa abdominal, sangramento intestinal, anemia e emagrecimento. O tratamento da mucocele é fundamentalmente cirúrgico e a complicação mais importante é o pseudomixoma peritoneal. Neste trabalho realizamos uma revisão literária e a descrição de dois casos sintomáticos de mucocele benigna do apêndice, diagnosticados e tratados no Hospital Escola Luiz Gioseffi Jannuzzi da Faculdade de Medicina de Valença (HELGJ-FMV), onde as pacientes evoluíram bem somente após a remoção cirúrgica do apêndice.
\end{abstract}

Palavras-chave: Mucocele, Apêndice, Laparotomia, Apendicectomia

\begin{abstract}
The appendix mucocele occurs after its obstruction when there is accumulation of mucinous material in its interior, thus dilating its light. It is a rare and usually asymptomatic disease, most often an incidental finding at the time of laparotomy. However, they may have abdominal pain, changes in bowel habits, abdominal mass, intestinal bleeding, anemia, and weight loss. The treatment of mucocele is primarily surgical and the most important complication is peritoneal pseudomyxoma. In this work we performed a literary review and the description of two symptomatic cases of benign mucocele of the appendix diagnosed and treated at the Hospital Escola Luiz Gioseffi Jannuzzi of the Faculdade de Medicina de Valença (HELGJ-FMV), where the patients evolved well only after the surgical removal of the appendix.

Keywords: Mucocele, Appendix, Laparotomy, Appendectomy
\end{abstract}

\section{Introdução}

A dilatação da luz do apêndice cecal, que ocorre após sua obstrução devido ao acúmulo de material mucinoso em seu interior, é denominada mucocele de apêndice. Essas alterações também levam ao adelgaçamento da parede apendicular e formação de cisto com acúmulo de conteúdo em seu interior ${ }^{1,4,13-15}$.

Foi descrita inicialmente em 1842 por Rokitansky e formalmente denominada por Feren em 1876,1,4,13,15-17 e consiste em uma doença rara, sendo observada em $0,07 \%$ a $0,7 \%$ das apendicectomias ${ }^{1-21}$. Corresponde à $8 \%$ de todos os tumores do apêndice ${ }^{5,7,15,17,21-23}$, predomina no sexo feminino, mais comumente na faixa etária acima de $50 \operatorname{anos}^{1-6,8,12-19}$.

Pode causar dores abdominais agudas ou crônicas, de padrão variável, localizadas em quadrante inferior direito, por vezes associada à massa palpável em fossa ilíaca direita e perda de peso. Entretanto, é uma doença assintomática em cerca de $25 \%$ dos $\operatorname{cas}^{1-5,8,9,11,13-20}$, sendo, na maioria das vezes, um achado incidental no momento da laparotomia. Exames de imagem e a dosagem de marcadores como os antígenos carcinoembrionário (CEA) e carboidrato (CA) 50 podem auxiliar o diagnóstico $1-11,13,14,17,18,21$.

O tratamento da mucocele de apêndice é cirúrgico, através da apendicectomia ou hemicolectomia direita. ${ }^{1-21}$ As complicações são raras, mas cita-se a intussuscepção, sangramento, perfuração, peritonite ${ }^{3,14,16,21}$, e a mais importante delas, o pseudomixoma peritoneal, quando de ruptura do apêndice, potencialmente fatal e mais comum em casos de mucocele de apêndice maligna ${ }^{3,8,12-20,22}$.

O objetivo deste trabalho foi relatar dois casos de mucocele benigna do apêndice diagnosticados e tratados no Hospital Escola Luiz Gioseffi Jannuzzi

Afiliação dos autores: $\uparrow$ Faculdade de Medicina do Centro de Ensino Superior de Valença (CESVA) - Fundação Dom André Arcoverde (FAA), Valença, RJł Xxxxxx

$\S$ Hospital Escola Luiz Gioseffi Jannuzzi (HELGJ) - Fundação Dom André Arcoverde (FAA), Valença, RJ

\| Liga Acadêmica de Cirurgia (LAC) - Fundação de Medicina de Valença (FAA), Valença, RJ

* Email para correspondencia: rrbtv@hotmail.com 
da Faculdade de Medicina de Valença (HELGJ-FMV) e fazer uma revisão de literatura sobre o assunto. Foi submetido projeto à plataforma Brasil, sendo aprovado com número de parecer 1.523.018. Procedeu-se então com busca de artigos científicos em periódicos indexados ao PubMed, LILACS e Scielo. Foram selecionados aqueles de maior relevância na construção deste trabalho e, após isso, os dados da literatura foram confrontados com os achados clínicos dos casos e apresentados na discussão.

\section{Relato de Caso 1}

Paciente do sexo feminino, 44 anos, branca, deu entrada no HELGJ relatando início de epigastralgia em cólica, com irradiação para hipocôndrio direito e dorso, sem outros sintomas associados. Posteriormente, além da dor abdominal, apresentou náuseas, diminuição do apetite e perda ponderal de $10 \mathrm{~kg}$, sem alteração do hábito intestinal. Dois meses após o início do quadro, apresentou piora da dor abdominal, agora difusa, associada à diarreia aquosa, vômitos biliosos e distensão abdominal.

Foi submetida a exames laboratoriais e de imagem. A ultrassonografia (USG) de abdome total evidenciou nefrolitíase à direita e pequena quantidade de líquido livre na cavidade abdominal. Tomografia computadorizada (TC) abdominal demonstrou também microcálculo em rim direito; além de distensão de alças intestinais de delgado com níveis hidroaéreos, espessamento parietal e dilatação dos cólons ascendente e transverso, sugerindo invaginação íleo-ceco-cólica. Colonoscopia mostrou volumosa lesão elevada ao nível do ceco, na submucosa, sugestiva de leiomioma ou lipoma.

Após preparo de cólon com manitol, foi submetida à incisão paramediana pararretal externa infraumbilical (incisão de Jalaguier) e encontrado apêndice aumentado de tamanho, sem sinais inflamatórios e com presença de massa endurecida na base junto ao ceco. Foi realizado apendicectomia e, com a peça aberta, constatou-se a presença de muco no interior, corroborando com diagnóstico de mucocele de apêndice. Após isso, a paciente evoluiu satisfatoriamente bem, sem mais queixas ou intercorrências.

\section{Relato de Caso 2}

Paciente do sexo feminino, 57 anos, branca, deu entrada no HELGJ relatando dor em fossa ilíaca direita em pontada, intermitente e iniciada há nove meses, sem outros sintomas associados. Foi conduzida ambulatorialmente com sintomáticos e encaminhada aos especialistas. Cinco meses depois, exame ginecológico revelou tumoração em topografia de ovário direito.

USG pélvica e transvaginal foram solicitadas e mostravam imagem cística de conteúdo anecóico medindo $39 \times 27 \times 27 \mathrm{~mm}$ em região anexial direita, sugerindo de cisto ovariano ou outra doença anexial. Exames laboratoriais evidenciaram $\mathrm{CEA}=3,23 \mathrm{U} /$ $\mathrm{mL}$ e CA $125=8,3 \mathrm{U} / \mathrm{mL}$. Foi solicitada nova USG transvaginal, agora com doppler colorido, que evidenciou ausência de ovário direito e uma formação predominantemente cística em sua topografia, de parede levemente espessada e irregular, apresentando área sólida em sua porção inferior, medindo $4,1 \times 2,5 \mathrm{~cm}$; sem vascularização intramural ou capsular.

Indicado laparotomia para realização de ooforectomia direita por incisão de Pfannenstiel. No ato operatório, foi evidenciado apêndice aumentado de tamanho, edemaciado, com ponto de perfuração onde se observava presença de substância gelatinosa (Figura
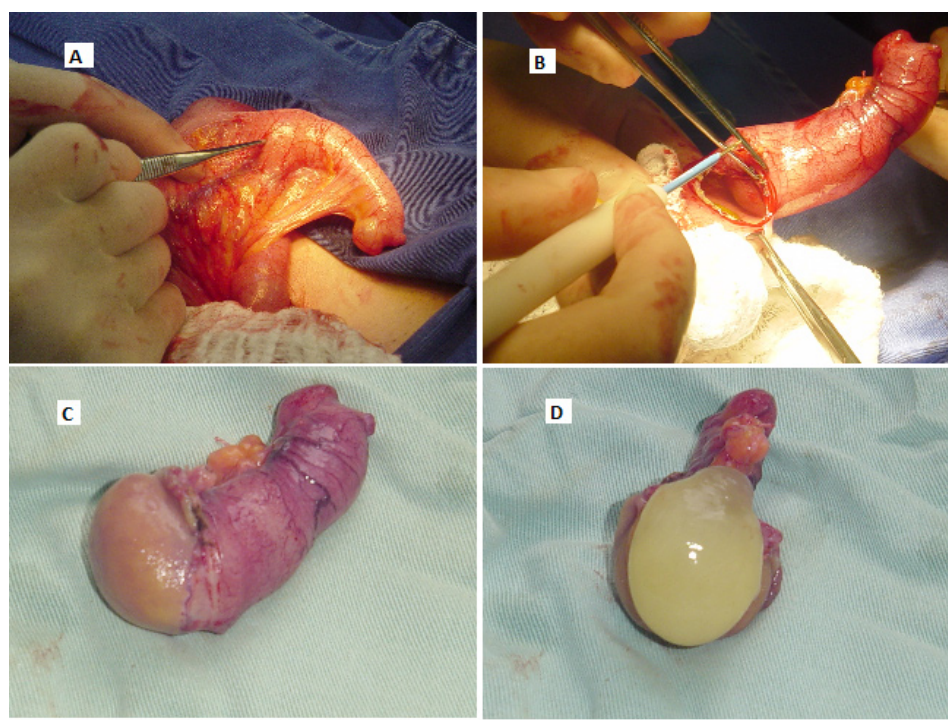

Figura 1. A-Achado cirúrgico. B - Procedimento cirúrgico. C - Peça cirúrgica fechada. D - Peça cirúrgica aberta (autoria própria) 


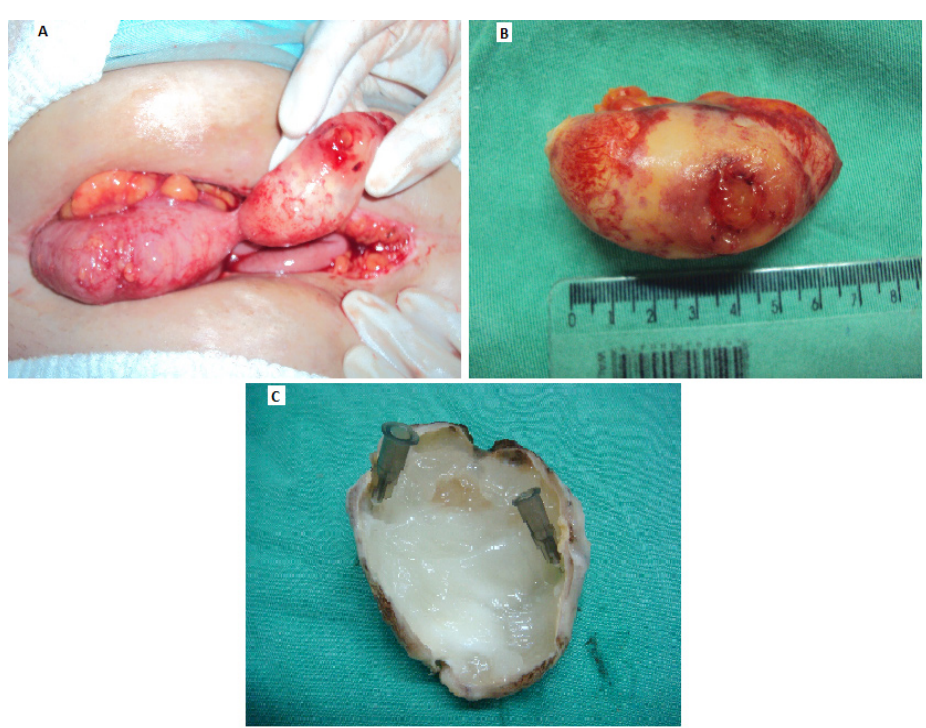

Figura 2. A - Achado operatório. B - Peça cirúrgica fechada. C - Peça cirúrgica aberta (autoria própria).

2 A); pelve sem implantes; útero e anexos normais. Foi realizado apendicectomia (Figura 2 B) e a peça foi aberta, constatando-se a presença de muco em seu interior (Figura $2 \mathrm{C}$ ).

A paciente evoluiu bem e recebeu alta no $3^{\circ}$ dia de pós-operatório. Apresentou seroma de ferida operatória e foi tratada com punção local, sem outras complicações. O exame anatomopatológico revelou mucocele de apêndice sem sinais de malignidade.

\section{Discussão}

A mucocele do apêndice, definida como um apêndice dilatado e cheio de muco, é uma entidade rara, que predomina em mulheres acima dos 50 anos $^{1-}$ 5,7-10,12-20. O tumor mais comum do apêndice é o tumor carcinoide, seguido do adenocarcinoma ${ }^{9,19}$.

Tendo sua origem inicialmente creditada à obstrução ou inflamação da luz apendicular pela presença de fecalitos, sabe-se hoje que o acúmulo mucoide, de forma lenta e gradual, é na verdade de origem neoplásica em sua maioria ${ }^{12}$.

As mucoceles de apêndice foram inicialmente classificadas em 1940 por Woodruff e McDonald como benignas ou malignas e divididas em quatro categorias clínico-patológicas 1,3,5,8,10-14,16-19,22. Todavia, posteriormente Higa et al (1973) classificaram as

Tabela 1. Características dos tipos de mucocele do apêndice segundo cada classificação.

\begin{tabular}{|c|c|c|}
\hline Tipos & Higa et al. (1973) & Woodruff e Mc Donald (1940) \\
\hline I & $\begin{array}{c}\text { Mucocele simples ou } \\
\text { cisto de retenção com hiperplasia } \\
\text { da mucosa, focal ou difusa, sem } \\
\text { atipia epitelial }\end{array}$ & $\begin{array}{c}\text { Mucocele simples ou cisto de } \\
\text { retenção }\end{array}$ \\
\hline II & $\begin{array}{l}\text { Cistoadenoma mucinoso } \\
\text { com algum grau de atipia epitelial; } \\
\text { tipo mais comum de mucocele }\end{array}$ & $\begin{array}{l}\text { Hiperplasia focal ou difusa da mucosa } \\
\text { do apêndice, sem atipia celular da mucosa e } \\
\text { com achados histológicos semelhantes aos } \\
\text { pólipos hiperplásicos do cólon }\end{array}$ \\
\hline III & $\begin{array}{l}\text { Cistoadenocarcinoma } \\
\text { mucinoso; existe invasão estromal } \\
\text { por glândulas neoplásicas ou } \\
\text { implantes peritoneais }\end{array}$ & $\begin{array}{l}\text { Cistoadenoma mucinoso, com } \\
\text { atipia celular da mucosa e com achados } \\
\text { histológicos semelhantes aos pólipos } \\
\text { adenomatosos e tumores vilosos; tipo mais } \\
\text { comum de mucocele }\end{array}$ \\
\hline
\end{tabular}


mucoceles de apêndice em apenas três categorias clínico-patológicas ${ }^{3,5,12,23}$. As principais diferenças entre estas duas classificações são descritas a seguir na Tabela 1.

Aproximadamente $25 \%$ dos pacientes são assintomáticos ${ }^{1,3-6,10,12-15,17-20}$, sendo o sintoma mais comum a dor abdominal aguda ou crônica em quadrante inferior direito, podendo ser observados com menor frequência alteração do hábito intestinal, sangramento intestinal, anemia, emagrecimento e massa abdominal $1^{1,3-6,8-19}$. Lesões que causam sintomas mais exuberantes estão mais associadas com mucocele maligna que as assintomáticas ${ }^{3,9,12,17}$, assim como em casos de mucoceles com diâmetro maior que 2 $\mathrm{cm}^{9,10,12}$.

Em $60 \%$ dos casos o diagnóstico é estabelecido no ato cirúrgico de laparotomias exploradoras ou pelo exame histopatológico ${ }^{1,3-5,7-10,12-14,16-19}$, ocorrendo também durante procedimentos laparoscópicos ${ }^{7}$. No entanto, exames complementares como USG e TC de abdome, clister opaco e colonoscopia, quando solicitados, podem sugerir o diagnóstico e auxiliar na condução da cirurgia ${ }^{1-6,8-13,15-21}$. O aprimoramento dos métodos de imagem tornou o diagnóstico de mucocele de apêndice possível antes da cirurgia, sendo a TC de abdome o melhor exame para estabelecer o diagnóstico e estimar a extensão da doença. A colonoscopia pode auxiliar mostrando massa eritematosa com uma cratera central (Sinal do Vulcão), que corresponde ao óstio do apêndice ${ }^{15,17}$.

Osdiagnósticosdiferenciais damucoceleincluem hidrossalpingite, cisto mesentérico, apendicite aguda, cistos de ovário e renal, dentre outros ${ }^{1,2,5,12,14,16,19,20}$.

É relatada na literatura a associação da mucocele com adenocarcinoma de cólon em um a cada cinco casos, enquanto que com cistoadenoma e cistoadenocarcinoma de ovário chega a $13 \% \%^{3,5,8,9,11-18}$. Neste contexto, a utilização de marcadores de doenças tumorais pode auxiliar na identificação de neoplasias de cólon ou ovário associadas à mucocele do apêndice, destacando-se a dosagem dos antígenos CEA e CA $50^{3,8,12,13,17,19}$. Também podemos encontrar associação com neoplasias de vesícula biliar, endométrio, mama, próstata, bexiga, rim $^{3,9,12,14}$ e estômago ${ }^{15}$.

O tratamento da mucocele do apêndice é cirúrgico $^{1-20}$, sendo utilizadas como vias de acesso a laparotomia ${ }^{1-4,8,10-18,20,21}$ e a videolaparoscopia ${ }^{7,9,13-}$ 18,20,21 . O acesso videolaparoscópico é contra indicado por muitos autores diante do risco aumentado de perfuração, extravasamento de muco para a cavidade abdominal e disseminação de implantes neoplásicos 3,8,12-17. Todavia, Palanivelu et al (2008) descrevem que o acesso videolaparoscópico para o tratamento de tumores apendiculares é seguro, viável e benéfico para o paciente ${ }^{7}$.

Quanto ao melhor procedimento indicado, se é a apendicectomia ou a hemicolectomia direita, a literatura é controversa, sendo que, independentemente do procedimento realizado, o cirurgião deve sempre garantir que não extravase conteúdo da mucocele para a cavidade abdominal, evitando assim o desenvolvimento de pseudomixoma peritoneal ${ }^{3-5,11-13,17,18}$. A escolha da apendicectomia, segundo alguns autores, está indicada em casos de mucocele benigna ${ }^{1,3,5,8-11,13-20}$. A hemicolectomia direita, segundo outros autores, deve ser a única indicação e a forma padrão de tratamento ${ }^{2,12-}$ ${ }^{18}$ ou quando a mucocele é maligna ${ }^{5,9,11,13,14,16-20}$. Para alguns autores, a hemicolectomia direita depende ainda de certos fatores ${ }^{3,7,9,15,17}$, destacando-se:

1. Necessidade de ressecção de toda a lesão;

2. Mucoceles maiores que $2 \mathrm{~cm}$ de diâmetro;

3. Acometimento linfonodal de mesoapêndice ou ileocólico ao histopatológico;

4. Diagnóstico de lesão maligna do apêndice do tipo não mucinoso.

Quando a lesão envolve a base do apêndice, sem acometimento linfonodal regional, o tratamento devese basear somente em ressecção do $\operatorname{ceco}^{3,4,11,17,21}$.

Pode-se ainda recorrer ao exame histopatológico de congelação das margens de ressecção quando há duvidas no acometimento da base apendicular ${ }^{3}$.

As complicações da mucocele de apêndice geralmente são raras e incluem hemorragias, intussuscepção,perfuraçãoe peritonite. Todavia, quando não adequadamente tratada, pode levar à perfuração e ruptura da luz apendicular, havendo extravasamento do conteúdo mucinoso repleto de células neoplásicas para a cavidade abdominal, complicação conhecida como pseudomixoma peritoneal. O rompimento pode ser espontâneo ou iatrogênico. É a complicação mais grave e de pior prognóstico, podendo ocorrer em mucoceles benignas ou malignas, apesar de intimamente relacionadas às últimas. Em seu curso, pode haver completa distensão abdominal devido ao preenchimento do peritônio com conteúdo adesivo e semissólido (depósitos de mucina), levando a obstrução intestinal, fistulação e potencialmente óbito por inanição. Sua resolução, quando possível, é obtida através de cirurgia peritoneal radical e quimioterapia local e sistêmica ${ }^{3,8,12-20}$.

Em pacientes com mucoceles benignas ressecadas, a sobrevida varia de $91 \%$ a $100 \%$ em cinco anos, inclusive mediante envolvimento extraapendicular. Já em mucoceles malignas, a sobrevida em cinco anos é muito reduzida, sendo $25 \%$ a $45 \%$ dos casos provenientes da evolução do pseudomixoma peritoneal $^{3,12,15,19,20}$.

Amortalidadeduranteoprocedimento operatório, principalmente devido a cistoadenocarcinoma e pseudomixoma peritoneal, pode variar de $4,8 \%$ a 
$22 \%{ }^{14,16}$

No primeiro caso aqui descrito, como o apêndice estava íntegro e não havia linfonodomegalia mesentérica, o ato cirúrgico transcorreu sem ruptura do órgão para a cavidade abdominal e não foram encontrados implantes peritoneais; optamos assim pela apendicectomia com ressecção limitada a uma cunha elíptica da parede do ceco, assegurando margens cirúrgicas livres. Já no segundo caso, foi visualizado ponto de perfuração no apêndice e o órgão completamente removido.

\section{Considerações finais}

A mucocele do apêndice é uma doença rara, normalmente com diagnóstico estabelecido no intraoperatório de laparotomias indicadas para o diagnóstico ou tratamento de outras doenças. Pode cursar com formas mais graves, como o pseudomixoma peritoneal, demandando uma investigação correta e tratamento imediato, o qual pode ser realizado através da apendicectomia ou hemicolectomia direita ${ }^{1,3,12}$.

No presente trabalho, foi apresentada uma revisão bibliográfica a propósito de dois casos de mucocele benigna do apêndice abordados no HELGJ-FMV. Os dois ocorridos em mulheres, corroborando com a literatura, porém, a primeira com menos de 50 anos no momento do diagnóstico, em desacordo com a faixa etária descrita na literatura; enquanto a segunda estava com 57 anos, dentro da faixa etária mais comum. Em nenhum dos casos as pacientes eram assintomáticas, apresentando em comum quadro de dor abdominal crônica.

A paciente do primeiro caso evoluiu ainda com inapetência, perda ponderal, intensificação da dor abdominal, distensão abdominal, náuseas e vômitos; foi submetida a exames complementares que sugeriam leiomioma ou lipoma. Já a paciente do segundo caso apresentou tumoração em topografia de ovário direito em exame ginecológico, compatível com cisto ovariano ou doença anexial pelos exames de imagem.

Nos dois casos aqui apresentados, o diagnóstico definitivo só foi possível no ato cirúrgico pela evidência de muco no interior do apêndice e as pacientes evoluíram bem somente após a apendicectomia.

\section{Referências Bibliográficas}

1. Andrade CF, Santo PRQE, Pantaroto M, Spadella CT. Cistoadenoma mucinoso de apêndice: relato de caso. Acta Cir Bras 2001;16(4):251-4.

2. Butte JMB, Torres JM, Arriagada IJ, Bustamante CR, Martinez JC. Appendiceal mucocele. Rev Chil Cir 2007;59(2):99-100.

3. Fonseca LM, Sassine GCA, Luz MMP, Silva RG, Conceição SA, LacerdaFilho A. Cistoadenoma de apêndice: relato de caso e revisão da literatura sobre tumores mucinosos do apêndice vermiforme. Rev Bras Coloproct 2008;28(1):89-94

4. Gonzalez Valverde EM, Gómez Ramos MJ, Vazquez Rojas JL. Extrinsic ileal occlusion secondary to appendiceal mucocele of unusual size. Acta Chir Belg 2008;108(4):454-6.

5. Guimarães Filho A, Magalhães JP, Campos ES, Guimarães JF, Alves Jr JL. Mucocele do apêndice. Rev Col Bras Cir 2008;35(1):64-5.

6. Karakaya K, Barut F, Emre AU, Ucan HB, Cakmak GK, Irkorucu O, Tascilar O, Ustundag Y, Comert M. Appendiceal mucocele: case reports and review of current literature. World J Gastroenterol 2008;14(4):2280-3.

7. Palanivelu C, Rangarajan M, John SJ, Senthilkumar K, Annapoorni S. Laparoscopic right hemicolectomy for mucocele due to a low-grade appendiceal mucinous neoplasm. JSLS 2008;12(2):194-7.

8. Pereira JCR, Silva Jr CA. Mucocele do apêndice. Rev Col Bras Cir 2008;28(3):225-7.

9. Premoli G, Pierini L, Ramos R, Minatti W, Capelino P. El mucocele apendicular. Revista del Hospital Privado de Comunidad 2003;6(1):1-2.

10. Ribeiro Filho EE, Mendes MBP, Castro EG, Tamura S, Macedo JLS, Lino EH, Sales PC. Mucocele gigante do apêndice: relato de caso. Rev Bras Coloproct 1997;17(1):44-6.

11. Ruiz-Tovar J, Turuel DG, Castiñeiras VM, Dehesa AS, Quindós PL, Molina EM. Mucocele of the appendix. World J Surg 2007;31(3):542-8.

12. Yamane YD, Yamane H, Castro Jr PC, Marsilac A, Mesquita RB, Lopes Paulo F. Mucocele do apêndice: relato de caso e revisão de literatura. Rev Bras Coloproct 2005;25(3):256-60.

13. Costa RGF. Mucocele de apêndice. Rev Col Bras Cir 2009;36(2):180-2.

14. Oliveira CC, Ferreira PRC. Cistoadenoma mucinoso de apêndice cecal: Relato de caso. Rev Paranaen Med 2015;29(1):51-5.

15. Polotto PPLS, Garcia MT, Gazola NF, Cretelli OS, Borim AA, Campos Jr E. Mucocele de apêndice vermiforme em paciente com câncer gástrico. Arq Ciênc Saúde 2014;21(1):66-70.

16. Martínez ACU, Soriano JAM, del Val Gil JM, Conde AC, Peris PL, Penabad MG, Gómez MS, Muzas CM. Mucocele apendicular. Rev Esp Enferm Dig 2008;100(11):730-8.

17. Abuoğlu H, Yildiz MK, Kaya B, Odabaşı. Clinicopathological analysis of patients operated for appendiceal mucocele. Ulus Travma Acil Cerrahi Derg, May 2017; 23(3):230-4.

18. Abdelhadi YS, Elhaj AAM. Appendiceal mucocele: benign and malignant. Sudan Med J April 2013;49(1):44-9.

19. Rymer B, Forsythe RO, Husada G. Mucocoele and mucinous tumours of the appendix: A review of the literature. International Journal of Surgery 2015;18:132-5

20. Lorenzon L, Dominicis C, Virgilio E., Balducci G. The appropriate management of an appendiceal mucocele. BMJ Case Rep 2015:1-3.

21. Stark C, Jousi M, Enholm B. Preoperative assessment and treatment of appendiceal mucocele complicated by acute torsion: a case report. BMC Research Notes 2014;7(1):1-5.

22. Woodruff R, McDonald JR. Benign and malignant cystic tumors of the appendix. Surg Gynecol Obstet. 1940;71:750-5.

23. Higa E, Rosai J, Pizzimbono CA, et al. Mucosal hyperplasia, mucinous cystoadenoma, and mucinous cystoadenocarcinoma of the appendix: A reevaluation of appendiceal mucocele. Cancer 1973;32:1525-41. 